\title{
A multiscale tumor model
}

\author{
AVNER FRIEDMAN ${ }^{\dagger}$ \\ Mathematical Biosciences Institute, The Ohio State University, \\ Columbus, $\mathrm{OH} 43210$, USA
}

[Received 15 May 2007 and in revised form 30 November 2007]

\begin{abstract}
We consider a tumor model with two time scales: the time $t$ during which the tumor evolves and the running time $s_{i}$ for each of the phases of the cell cycle for the cells in the tumor. The model also includes the effect of genes mutations in the sense that populations of cells with different mutations and in different phases of the cell cycle evolve by different rules. The model is formulated as a coupled system of partial differential equations; a transition from one population to another occurs at the 'restriction points' located at the ends of the $G_{1}$ and $S$ phases. The PDEs for the cell populations are hyperbolic equations based on mass conservation laws. The model includes also a diffusion equation for the oxygen concentration and an elliptic equation for the internal pressure caused by proliferation and death of cells. The tumor region is viewed as a domain with a moving boundary, satisfying a continuity equation at the free boundary. Existence and uniqueness are proved for a small time interval, for general initial conditions, and for all time in the case of radially symmetric initial conditions.
\end{abstract}

\section{Introduction}

The cell cycle is divided into phases $G_{1}, S, G_{2}$ and $M$. During the $S$ phase the DNA is synthesized; during the mitosis phase $M$ sister chromosomes are segregated as the cell prepares to divide into two daughter cells; $G_{1}$ and $G_{2}$ are 'gap' phases, during which the cell grows and prepares for the next phase ( $S$ for $G_{1}$, and $M$ for $G_{2}$ ). At a 'restriction point' $R$ located near the end of the $G_{1}$ phase the cell decides either to proceed directly to the $S$ phase, or to go into quiescent state $G_{0}$, depending on the environment; the cell may also decide to go into apoptosis (i.e., to commit suicide) in case it detects serious damage. At another restriction point, located at the end of the $S$ phase, the cell again has to make a decision: whether to proceed to the $G_{2}$ phase or to go into apoptosis, in case it detects damage. A cell remains in state $G_{0}$ for a certain amount of time and then proceeds to the $S$ phase.

At the restriction point $R$ the cell checks the environment for signals of hypoxia, overpopulation, etc. Specific genes detect hypoxia and overpopulation signals. When these genes are mutated, the cell may continue to proliferate uncontrollably and a tumor will develop. For example, one of the first genes whose mutation is associated with colorectal cancer is APC. This gene detects a signal of overpopulation and it then inhibits proliferation by sending the cell into the $G_{0}$ state. Another gene, SMAD, is activated after receiving hypoxia signals and it then inhibits proliferation, again by sending the cell into the $G_{0}$ state. If these genes are mutated, the cell ignores overpopulation and hypoxia signals and this leads to uncontrollable cell division and the development of a tumor; for more details see [24]. The gene APC is believed to be the primary gene in the initiation of a colorectal tumor; other mutations subsequently develop.

\footnotetext{
${ }^{\dagger}$ E-mail: afriedman@mbi.osu.edu
} 
In this paper, we model tumor growth by distinguishing between populations of cells according to their phase in the cell cycle and according to their mutations. For simplicity, we consider cells with at most one mutation (e.g., only APC mutation in the case of colorectal cancer), but the analysis easily extends to any number of mutations. We introduce, in addition to the absolute time $t$, the time $s_{i}$ for cells in the $i$-th phase. Our model is multiscale in the sense that it deals with two time scales ( $t$ and $s_{i}$ ) and in the sense that it incorporates (molecular) DNA events with (macroscopic) cell populations dynamics.

We consider first populations of cells with no mutations, and introduce the following notation:

$$
\begin{aligned}
p_{1}\left(x, t, s_{1}\right) & =\text { density of cells in phase } G_{1}, s_{1} \in K_{1} \equiv\left[0, A_{1}\right] \\
p_{2}\left(x, t, s_{2}\right) & =\text { density of cells in phase } S, s_{2} \in K_{2} \equiv\left[0, A_{2}\right] \\
p_{0}\left(x, t, s_{0}\right) & =\text { density of cells in state } G_{0}, s_{0} \in K_{0} \equiv\left[0, A_{0}\right] \\
p_{3}\left(x, t, s_{3}\right) & =\text { density of cells in phases } G_{2} \cup M, s_{3} \in K_{3} \equiv\left[0, A_{3}\right] ; \\
p_{4}(x, t) & =\text { density of necrotic cells. }
\end{aligned}
$$

The variable $x$ will vary in the tumor region $\Omega(t)$ in $\mathbb{R}^{N}(N \geqslant 2)$ with boundary $\Gamma(t)$.

We denote by $w(x, t)$ the oxygen concentration and by $Q(x, t)$ the density of live cells. Due to cell proliferation and death, there is a velocity field $\vec{v}(x, t)$, which is assumed to be common to all the cells. Then, by conservation of mass,

$$
\begin{aligned}
\frac{\partial p_{i}}{\partial t}+\frac{\partial p_{i}}{\partial s_{i}}+\operatorname{div}\left(p_{i} \vec{v}\right) & =\lambda_{i}(w) p_{i} \quad \text { for } 0<s_{i}<A_{i} \quad(i=0,1,2,3), \\
\frac{\partial p_{4}}{\partial t}+\operatorname{div}\left(p_{4} \vec{v}\right) & =\mu_{1} p_{1}\left(x, t, A_{1}\right)+\mu_{2} p_{2}\left(x, t, A_{2}\right)-\lambda_{4} p_{4}
\end{aligned}
$$

where $\lambda_{i}(w)$ are growth rates, which depend on the oxygen concentration $w, \lambda_{4}$ is the clearing rate of dead cells, and $\mu_{1}, \mu_{2}$ are the rates at which cells at the endpoints of $G_{1}$ and $S$ go into apoptosis.

We also have:

$$
\begin{aligned}
& p_{1}(x, t, 0)=p_{3}\left(x, t, A_{3}\right), \\
& p_{2}(x, t, 0)=p_{1}\left(x, t, A_{1}\right)\left[1-K(w(x, t))-L(Q(x, t))-\mu_{1}\right]+p_{0}\left(x, t, A_{0}\right), \\
& p_{3}(x, t, 0)=\left(1-\mu_{2}\right) p_{2}\left(x, t, A_{2}\right), \\
& p_{0}(x, t, 0)=p_{1}\left(x, t, A_{1}\right)[K(w(x, t))+L(Q(x, t))] .
\end{aligned}
$$

The second equation in (2) expresses the assumption that at the end of the $G_{1}$ phase a fraction $K(w)+L(Q)$ of the cells go into quiescence, and a fraction $\mu_{1}$ go into apoptosis, while the cells at the end of the quiescence period enter the $S$ phase. Naturally we assume that

$$
\begin{gathered}
K(w)>0, \quad L(Q)>0, \quad K(w)+L(Q)+\mu_{1}<1, \\
K(w) \downarrow \quad \text { if } w \uparrow \quad \text { and } \quad L(Q) \downarrow \quad \text { if } Q \downarrow .
\end{gathered}
$$

Suppose next that the tumor cells underwent only APC mutation, and introduce, analogously to $p_{i}\left(x, t, s_{i}\right)$, densities of mutated cells $p_{i}^{a}\left(x, t, s_{i}\right)$ where $s_{i} \in K_{i}^{a}$, and $K_{i}^{a} \equiv\left[0, A_{i}^{a}\right], 0 \leqslant i \leqslant 3$; the density of the dead cell will be denoted by $p_{4}^{a}(x, t)$. Then, analogously to $(1)$, we have

$$
\begin{aligned}
\frac{\partial p_{i}^{a}}{\partial t}+\frac{\partial p_{i}^{a}}{\partial s_{i}}+\operatorname{div}\left(p_{i}^{a} \vec{v}\right) & =\lambda_{i}^{a}(w) p_{i}^{a} \quad \text { for } 0<s_{i}<A_{i}^{a}(i=0,1,2,3) \\
\frac{\partial p_{4}^{a}}{\partial t}+\operatorname{div}\left(p_{4}^{a} \vec{v}\right) & =\mu_{1}^{a} p_{1}^{a}\left(x, t, A_{1}^{a}\right)+\mu_{2}^{a} p_{2}^{a}\left(x, t, A_{2}^{a}\right)-\lambda_{4}^{a} p_{4}^{a}
\end{aligned}
$$


and analogously to (2) we have

$$
\begin{aligned}
& p_{1}^{a}(x, t, 0)=p_{2}^{a}\left(x, t, A_{3}^{a}\right), \\
& p_{2}^{a}(x, t, 0)=p_{1}^{a}\left(x, t, A_{1}^{a}\right)\left[1-K(w(x, t))-\mu_{1}^{a}\right]+p_{0}^{a}\left(x, t, A_{0}^{a}\right), \\
& p_{3}^{a}(x, t, 0)=\left(1-\mu_{2}^{a}\right) p_{2}^{a}\left(x, t, A_{2}^{a}\right), \\
& p_{0}^{a}(x, t, 0)=p_{1}^{a}\left(x, t, A_{1}^{a}\right) K(w(x, t)) .
\end{aligned}
$$

We introduce the total density of each population of cells:

$$
Q_{i}(x, t)=\int_{0}^{A_{i}} p_{i}\left(x, t, s_{i}\right) \mathrm{d} s_{i}, \quad Q_{i}^{a}(x, t)=\int_{0}^{A_{i}^{a}} p_{i}^{a}\left(x, t, s_{i}\right) \mathrm{d} s_{i}
$$

and set

$$
\vec{Q}=\left\{Q_{i}\right\}_{i=0}^{4}, \quad \vec{Q}^{a}=\left\{Q_{i}^{a}\right\}_{i=0}^{4}
$$

here we have formally set

$$
\begin{gathered}
p_{4}\left(x, t, s_{4}\right)=p_{4}(x, t), \quad p_{4}^{a}\left(x, t, s_{4}\right)=p_{4}^{a}(x, t), \\
s_{4} \in K_{4}=K_{4}^{a}=\left[0, A_{4}\right], \quad A_{4}=A_{4}^{a}=1 .
\end{gathered}
$$

The density of the live cells is given by

$$
Q=\sum_{i=0}^{3}\left(Q_{i}+Q_{i}^{a}\right)
$$

We integrate each of the equations in (1) over $s_{i} \in\left(0, A_{i}\right)$ and sum up the resulting equations. Using (2) we find that all the boundary integrals resulting from integrating $\partial p_{i} / \partial s_{i}$ cancel out, so that

$$
\sum_{i=0}^{4}\left[\frac{\partial Q_{i}}{\partial t}+\operatorname{div}\left(Q_{i} \vec{v}\right)\right]=\sum_{i=0}^{3} \lambda_{i}(w) Q_{i}-\lambda_{4} Q_{4}
$$

Similarly,

$$
\sum_{i=0}^{4}\left[\frac{\partial Q_{i}^{a}}{\partial t}+\operatorname{div}\left(Q_{i}^{a} \vec{v}\right)\right]=\sum_{i=0}^{3} \lambda_{i}^{a}(w) Q_{i}^{a}-\lambda_{4}^{a} Q_{4}^{a} .
$$

We assume that the tumor tissue is a porous medium satisfying Darcy's law

$$
\vec{v}=-\nabla \sigma
$$

where $\sigma$ is the pressure resulting from the movement of cells.

We also assume that the tumor tissue is packed uniformly by the cells, that is,

$$
\sum_{i=0}^{4}\left(Q_{i}+Q_{i}^{a}\right)=\text { const }=c_{0}
$$

and, for simplicity, take $c_{0}=1$. Then, by summing up (5), (6) we obtain

$$
\operatorname{div} \vec{v} \equiv-\nabla^{2} \sigma=H\left(\vec{Q}, \vec{Q}^{a}\right)
$$


where

$$
H\left(\vec{Q}, \vec{Q}^{a}, w\right)=\sum_{i=0}^{3}\left[\lambda_{i}(w) Q_{i}+\lambda_{i}^{a}(w) Q_{i}^{a}\right]-\left(\lambda_{4} Q_{4}+\lambda_{4}^{a} Q_{4}^{a}\right) .
$$

If we substitute (7) into (1) and (3) we obtain a system of the form

$$
\begin{gathered}
\frac{\partial p_{i}}{\partial t}+\frac{\partial p_{i}}{\partial s_{i}}-\nabla \sigma \cdot \nabla p_{i}=p_{i} f_{i}\left(\vec{Q}, \vec{Q}^{a}, w\right) \quad(0 \leqslant i \leqslant 3), \\
\frac{\partial p_{i}^{a}}{\partial t}+\frac{\partial p_{i}^{a}}{\partial s_{i}}-\nabla \sigma \cdot \nabla p_{i}^{a}=p_{i}^{a} f_{i}^{a}\left(\vec{Q}, \vec{Q}^{a}, w\right) \quad(0 \leqslant i \leqslant 3), \\
\frac{\partial p_{4}}{\partial t}-\nabla \sigma \cdot \nabla p_{4}=\mu_{1} p_{1}\left(x, t, A_{1}\right)+\mu_{2} p_{2}\left(x, t, A_{2}\right)+p_{4} f_{4}\left(\vec{Q}, \vec{Q}^{a}, w\right), \\
\left.\frac{\partial p_{4}^{a}}{\partial t}-\nabla \sigma \cdot \nabla p_{4}^{a}=\mu_{1}^{a} p_{1}^{a}\left(x, t, A_{1}^{a}\right)+\mu_{2}^{a} p_{2}^{a}\right)\left(x, t, A_{2}^{a}\right)+p_{4}^{a} f_{4}^{a}\left(\vec{Q}, \vec{Q}^{a}, w\right),
\end{gathered}
$$

where

$$
\begin{aligned}
& f_{i}\left(\vec{Q}, \vec{Q}^{a}, w\right)=\lambda_{i}(w)-H\left(\vec{Q}, \vec{Q}^{a}, w\right) \quad(0 \leqslant i \leqslant 3), \\
& f_{i}^{a}\left(\vec{Q}, \vec{Q}^{a}, w\right)=\lambda_{i}^{a}(w)-H\left(\vec{Q}, \vec{Q}^{a}, w\right) \quad(0 \leqslant i \leqslant 3), \\
& f_{4}\left(\vec{Q}, \vec{Q}_{a}, w\right)=-\lambda_{4}-H\left(\vec{Q}, \vec{Q}_{a}, w\right), \\
& f_{4}\left(\vec{Q}, \vec{Q}_{a}, w\right)=-\lambda_{4}^{a}-H\left(\vec{Q}, \vec{Q}_{a}, w\right) .
\end{aligned}
$$

Notice that the last equation in (9) is actually a consequence of the preceding equations in 9 ) and 77. We can therefore drop it provided we set $p_{4}^{a}=1-p_{4}-\sum_{i=0}^{3}\left(Q_{i}+Q_{i}^{a}\right)$ wherever $p_{4}^{a}$ appears. Finally, we assume that the oxygen concentration satisfies the diffusion equation

$$
w_{t}-D_{w} \nabla^{2} w+\lambda Q w=0
$$

where $D_{w}$ and $\lambda$ are positive constants.

We next prescribe boundary conditions at the free boundary $\Gamma(t)$ :

$$
\begin{array}{ll}
w=\bar{w} & \text { on } \Gamma(t), t>0, \\
\sigma=\lambda \kappa & \text { on } \Gamma(t), t>0
\end{array}
$$

where $\bar{w}, \lambda$ are positive constants and $\kappa$ is the mean curvature; $\kappa=1 / R$ if $\Gamma(t)$ is a sphere of radius $R$. We also impose the continuity assumption $\vec{v} \cdot \vec{n}=V_{n}$ where $V_{n}$ is the velocity of the free boundary in the outward normal direction $\vec{n}$, i.e.,

$$
V_{n}=-\frac{\partial \sigma}{\partial n}
$$

Finally, we prescribe initial conditions:

$$
\begin{aligned}
\left.\Omega(t)\right|_{t=0} & =\Omega_{0} & \text { is given, with boundary } \Gamma_{0}, & \\
\left.w\right|_{t=0} & =w_{0}(x) & & \text { for } x \in \Omega_{0}, \\
\left.p_{i}\right|_{t=0} & =p_{i_{0}}\left(x, s_{i}\right) & & \text { for } x \in \Omega_{0}, s_{i} \in K_{i}(0 \leqslant i \leqslant 4), \\
\left.p_{i}^{a}\right|_{t=0} & =p_{i_{0}}^{a}\left(x, s_{i}\right) & & \text { for } x \in \Omega_{0}, s_{i} \in K_{i}^{a}(0 \leqslant i \leqslant 4),
\end{aligned}
$$


and the $p_{i_{0}}, p_{i_{0}}^{a}$ satisfy the conditions

$$
p_{i_{0}} \geqslant 0, \quad p_{i_{0}}^{a} \geqslant 0, \quad \text { and } \quad \sum_{i=0}^{4}\left(Q_{i}+Q_{i}^{a}\right) \equiv 1 \quad \text { at } t=0 .
$$

Some of equations in (17)-(15) follow from the others; for instance, (9) is just a reformulation of (17, 3). Nevertheless, for simplicity, we shall refer to the problem of solving for the $p_{i}, p_{i}^{a}, w, \vec{v}$ and $\Omega(t)$ as Problem (1)- (15).

In Section 4 we prove that Problem (11)-(15) has a unique solution for a small time interval. In Section 5 we consider the case of radially symmetric initial data and prove that there exists a globalin-time radially symmetric solution with free boundary $r=R(t)$. Sections 2 and 3 are preparations for proving the existence theorems.

REMARK 1.1 If $X=X(t)$ is a characteristic curve of the hyperbolic system (1), (3) and $X\left(t_{0}\right) \in$ $\Gamma\left(t_{0}\right)$, then

$$
\left.\frac{\mathrm{d} X}{\mathrm{~d} t}\right|_{t=t_{0}} \cdot \vec{n}=\vec{v}\left(X\left(t_{0}\right), t_{0}\right) \cdot \vec{n}=V_{n}
$$

where $\vec{n}$ is the outward normal to $\Gamma\left(t_{0}\right)$ at $X\left(t_{0}\right)$; hence a characteristic curve starting on the free boundary $\Gamma\left(t_{0}\right)$ will remain on the free $\Gamma(t)$ for all $t$. Consequently, we do not need to prescribe boundary conditions for $p_{i}\left(x, t, s_{i}\right), p_{i}^{a}\left(x, t, s_{i}\right)$ on the free boundary $\Gamma(t)$.

REMARK 1.2 A multiscale hybrid model for a colorectal tumor was recently introduced by Ribba et al. [24]. In this model, the $s_{i}$ is replaced by a finite number of time steps, and the model is considered in a fixed domain. Another multiscale tumor model in a fixed domain was recently introduced by Ayati et al. [2]. Their model includes diffusion of cells and haptotaxis.

REMARK 1.3 A mathematical model of tumor with three populations of cells, namely, proliferating, quiescent, and necrotic, was introduced and studied numerically in [23]; mathematical analysis of the model appeared in [10], [11], [13], [14]. A tumor model with just proliferating cells was studied by many authors; see [1], [4]-[6], [8], [9], [15], [17]-[20] and the references in the review article [16]. The boundary condition [12) first appeared in the work of Greenspan [21], [22], and the role of the cell-to-cell adhesion as represented by the parameter $\gamma$ was discussed by Byrne [3], [5] and Byrne and Chaplain [7].

\section{The main result}

DEFinition 2.1 If the conditions (2), (4) are satisfied at $t=0$ by the initial data (14), and if $w_{0}=\bar{w}$ on $\Gamma_{0}$, then we say that the first order compatibility conditions are satisfied.

Our goal is to prove that under these compatibility conditions the system (1)-15) has a unique solution for some time interval $0 \leqslant t \leqslant T$. In order to define the regularity class for the solution we need some notation. 
Let $\varphi=\varphi(x, t, s), \beta=\left(\beta_{1}, \ldots, \beta_{N}, \beta_{N+1}, \beta_{N+2}\right), \beta_{i}$ integers $\geqslant 0,|\beta|=\beta_{1}+\cdots+\beta_{N+2}$. Then we write

$$
\begin{gathered}
D^{\beta} \varphi=D_{(x, t, s)}^{\beta} \varphi=\frac{\partial^{|\beta|} \varphi}{\left(\partial x_{1}\right)^{\beta_{1}} \ldots\left(\partial x_{N}\right)^{\beta_{N}}(\partial t)^{\beta_{N+1}}(\partial s)^{\beta_{N+2}}} \\
\|\varphi\|_{0}=\sup |\varphi|, \quad\|\varphi\|_{m}=\sum_{|\beta| \leqslant m}\left\|D^{\beta} \varphi\right\|_{0} \\
|\varphi|_{\alpha_{1}, \alpha_{2}, \alpha_{3}}=\sup \frac{|\varphi(x, t, s)-\varphi(\bar{x}, \bar{t}, \bar{s})|}{|x-\bar{x}|^{\alpha_{1}}+|t-\bar{t}|^{\alpha_{2}}+|s-\bar{s}|^{\alpha_{3}}} \\
\|\varphi\|_{m+\alpha_{1}, m+\alpha_{2}, m+\alpha_{3}}=\|\varphi\|_{0}+\sum_{|\beta|=m}\left|D^{\beta} \varphi\right|_{\alpha_{1}, \alpha_{2}, \alpha_{3}}
\end{gathered}
$$

here $m$ is an integer $\geqslant 0$ and $0<\alpha_{i}<1$. The domain in which the norms are defined will be specified later on. If $\varphi$ does not depend on $s$, then we define the corresponding norms by dropping $\alpha_{3}$. If $\varphi=\varphi(x, t)$, we define, for $0<\alpha<1$,

$$
\|\varphi\|_{3+\alpha,(3+\alpha) / 3}=\|\varphi\|_{0}+\left\|D_{x}^{3} \varphi\right\|_{\alpha, \alpha / 3}+\left\|D_{t} \varphi\right\|_{\alpha, \alpha / 3},
$$

Note that the norm (16) dominates $\|\varphi\|_{m}$, and, by standard estimates, the norm (17) dominates

$$
\left|D_{x}^{2} \varphi\right|_{0,(1+\alpha) / 2}+\left|D_{x} \varphi\right|_{0,(2+\alpha) / 3}
$$

where $|\psi|_{0, \alpha}=\sup _{x}|\psi(x, \cdot)|_{\alpha}$ if $\psi=\psi(x, t)$.

We say that a function $\varphi=\varphi(x, t, s)$ is in $C^{m+\alpha_{1}, m+\alpha_{2}, m+\alpha_{3}}$ if

$$
\|\varphi\|_{m+\alpha_{1}, m+\alpha_{2}, m+\alpha_{3}}<\infty .
$$

Similarly we define the notion of $\varphi=\varphi(x, t)$ in $C^{3+\alpha,(3+\alpha) / 3}$.

In the following we assume that

$$
\Gamma_{0} \in C^{m+4+\alpha}
$$

where $0<\alpha<1$ and $m$ is an integer $\geqslant 0$. Denote by $\xi$ a variable point in $\Gamma_{0}$ and by $\vec{n}(\xi)$ the unit outward normal to $\Gamma_{0}$ at $\xi$. We shall write $\Gamma(t)$ in the form of [11], [12] where these coordinates are used and play an important role:

$$
\Gamma(t)=\{\xi+\rho(\xi, t) \vec{n}(\xi)\}
$$

Set $d=d(x)=d\left(x, \Gamma_{0}\right)=$ signed distance from $x$ to $\Gamma_{0}\left(d>0\right.$ if $\left.x \notin \Omega_{0}\right)$. Then for $x$ near $\Gamma_{0}$ we can write

$$
x=\xi+d \vec{n}(\xi)
$$

where $\xi$ is uniquely determined by $x$.

In what follows we shall use a local coordinate transformation to flatten the boundary $\Gamma(t)$. We fix a point $\xi_{0}$ in $\Gamma_{0}$ and take local coordinates $y^{\prime}=\left(y_{1}, \ldots, y_{n-1}\right)$ near the origin 0 in $\mathbb{R}^{N-1}$, about $\xi_{0}$, so that any point $\xi \in \Gamma_{0}$ with $\left|\xi-\xi_{0}\right|$ small can be written in the form $\xi=S\left(y^{\prime}\right)$. Then any point $x \in \mathbb{R}^{N}$ near $\xi_{0}$ can be written in the form

$$
x=S\left(y^{\prime}\right)+\left(\rho(\xi, t)+y_{N}\right) \vec{n}\left(S\left(y^{\prime}\right)\right)
$$


where $y_{N}=d\left(x, \Gamma_{0}\right)-\rho(\xi, t)$. This defines a local mapping $y \mapsto x$ from a neighborhood of the origin in $\mathbb{R}^{N}$ into an $\mathbb{R}^{N}$-neighborhood of $\xi_{0}$ such that $x \in \Gamma(t)$ corresponds to $\left(y^{\prime}, 0\right)$. Although the coordinates $\left(y^{\prime}, y_{N}\right)$ will not appear explicitly in the following, they do appear implicitly; indeed, we shall refer the reader from time to time to results from [11], [12] where these coordinates are used and play an important role.

Later on we shall make the following regularity assumptions:

$$
\begin{array}{r}
\lambda_{i}(z), \lambda_{i}^{a}(z), K(z) \text { and } L(z) \text { belong to } C^{m+1+\alpha}\left(\mathbb{R}^{1}\right), \\
w_{0} \in C^{m+1+\alpha}\left(\bar{\Omega}_{0}\right), \text { the } p_{i_{0}} \text { belong to } C^{m+1+\alpha}\left(\bar{\Omega}_{0} \times K_{i}\right), \\
\text { and the } p_{i_{0}}^{a} \text { belong to } C^{m+1+\alpha}\left(\bar{\Omega}_{0} \times K_{i}^{a}\right)
\end{array}
$$

where $m$ is an integer $\geqslant 0$.

We first consider the case $m=0$ and assume that

the first order compatibility conditions are satisfied.

THEOREM 1 Under the assumptions (18)-20) for $m=0$ and (21), there exists a unique solution of Problem (1)- 15 ) for some time interval $0 \leqslant t \leqslant T(T>0)$ such that

$$
D_{\xi} \rho \in C^{3+\alpha,(3+\alpha) / 3}\left(\Gamma_{0} \times[0, T]\right),
$$

and $\sigma, w, p_{i}, p_{i}^{a}$ can be extended to functions satisfying:

$$
\begin{aligned}
D_{x}^{2} \sigma \in C^{\alpha, \alpha / 3}\left(\mathbb{R}^{N} \times[0, T]\right), & w \in C^{2+\alpha, 1+\alpha / 3}\left(\mathbb{R}^{N} \times[0, T]\right), \\
p_{i} \in C^{1+\alpha, \alpha / 3, \alpha / 3}\left(\mathbb{R}^{N} \times[0, T] \times K_{i}\right), & p_{i}^{a} \in C^{1+\alpha, \alpha / 3, \alpha / 3}\left(\mathbb{R}^{N} \times[0, T] \times K_{i}^{a}\right) .
\end{aligned}
$$

The proof of Theorem 1 is given in Section 4; it uses auxiliary lemmas which are given in Section 3. At the end of Section 4 we shall briefly consider higher regularity of the solution, when [19], 20] hold with $m>0$.

REMARK 2.1 It will be convenient to extend the initial data $w_{0}, p_{i_{0}}, p_{i_{0}}^{a}$ from $x \in \Omega_{0}$ to $x \in \mathbb{R}^{N}$ so that they vanish if $|x|$ is sufficiently large and 20 holds with $\Omega_{0}$ replaced by $\mathbb{R}^{N}$. The existence and uniqueness assertions of Theorem 1 will be established for these extended initial data. Since, by Remark 1.1, characteristic curves $X=X(t)$ with $X\left(t_{0}\right) \in \Omega\left(t_{0}\right)$ do not leave $\Omega(t)$ for all $t \in[0, T]$ and characteristic curves with $X\left(t_{0}\right) \in \Gamma\left(t_{0}\right)$ will lie in $\Gamma(t)$ for all $\left.t \in[0, T]\right)$, the uniqueness part of Theorem 1 does not depend on the above extension of the initial data.

\section{Auxiliary lemmas}

The proof of Theorem 1 is based on two lemmas. The first one, taken from [11], is concerned with the inhomogeneous Hele-Shaw problem: Find a function $\sigma(x, t)$ and domains $\Omega(t)$ such that

$$
\begin{gathered}
\Delta \sigma=h(x, t) \quad \text { in } \Omega(t), 0 \leqslant t \leqslant T, \\
\sigma=\gamma \kappa, \quad V_{n}=-\frac{\partial \sigma}{\partial n} \quad \text { on } \Gamma(t), 0 \leqslant t \leqslant T,
\end{gathered}
$$

where $\Omega(0)=\Omega_{0}$ is given, $\Gamma_{0}=\partial \Omega_{0}$ is as in $(18)$, and

$$
h \in C^{m+\alpha, m+\alpha / 3}\left(\mathbb{R}^{N} \times\left[0, T_{0}\right]\right)
$$

for some $T_{0}>0$. 
LEMMA 3.1 Under the assumptions (18), (24) for some integer $m \geqslant 0$, there exists a unique solution of 22, 23) for some $0<T \leqslant T_{0}$, such that

$$
D_{\xi} D_{(\xi, t)}^{m} \rho \in C^{3+\alpha,(3+\alpha) / 3}\left(\Gamma_{0} \times[0, T]\right)
$$

and $\sigma$ can be extended to a function satisfying

$$
D_{x}^{2} D_{(x, t)}^{m} \sigma \in C^{\alpha, \alpha / 3}\left(\mathbb{R}^{N} \times[0, T]\right)
$$

furthermore, $T$ depends only on the $C^{m+4+\alpha}$ regularity of $\Gamma_{0}$, and

$$
\left\|D_{\xi} D_{(\xi, t)}^{m} \rho\right\|_{3+\alpha,(3+\alpha) / 3}+\left\|D_{x}^{2} D_{(x, t)}^{m} \sigma\right\|_{\alpha, \alpha / 3} \leqslant C\|h\|_{m+\alpha, m+\alpha / 3}
$$

where $C$ depends only on the $C^{m+4+\alpha}$ regularity of $\Gamma_{0}$.

In the last inequality the first norm on the left-hand side is taken over $\Gamma_{0} \times[0, T]$ and the second norm is taken over $\mathbb{R}^{N} \times[0, T]$.

Lemma 3.1 was briefly stated in [11]. Its proof follows very similarly to the proof of Theorem 1 in [12]; most specifically, one needs just to observe that the estimates of the model problem in [12] for the inhomogeneous system [22] of [12] immediately extend to the system [22], 23] with general $h$.

The second lemma is an extension of Lemma 2.2 of [11] to the case of two time variables. Consider the hyperbolic equation

$$
W_{t}+W_{s}+\vec{b}(x, t) \cdot \nabla_{x} W=G(x, t, s, W) \quad \text { for } x \in \mathbb{R}^{N}, 0<t<T, 0<s<A
$$

with initial conditions

$$
\begin{array}{ll}
\left.W\right|_{t=0}=W_{0}(x, s) & \text { for } x \in \mathbb{R}^{N}, 0 \leqslant s \leqslant A, \\
\left.W\right|_{s=0}=W_{1}(x, t) & \text { for } x \in \mathbb{R}^{N}, 0 \leqslant t \leqslant T
\end{array}
$$

satisfying the compatibility condition

$$
W_{0}(x, 0)=W_{1}(x, 0), \quad x \in \mathbb{R}^{N} .
$$

LEMMA 3.2 Assume that

$$
\begin{gathered}
\vec{b}, D_{x} \vec{b} \text { belong to } C^{\alpha_{1}, \alpha_{2}}\left(\mathbb{R}^{N} \times[0, T]\right) \\
G, D_{W} G \text { belong to } C^{\alpha_{1}, \alpha_{2}, \alpha_{2}}\left(\mathbb{R}^{N} \times[0, T] \times[0, A]\right) \\
\text { and } D_{x} G, D_{s} G \text { belong to } C^{\alpha_{1}, \alpha_{2}, \alpha_{2}}\left(N_{0}\right) \\
\text { for any } W=W(x, t, s) \text { in } C^{\alpha_{1}, \alpha_{2}, \alpha_{2}}\left(\mathbb{R}^{N} \times[0, T] \times[0, A]\right)
\end{gathered}
$$

where $N_{0}$ is the disjoint union

$$
\begin{gathered}
\mathbb{R}^{N} \times[0, T] \times[0, A] \cap\{t<s\} \cup \mathbb{R}^{N} \times[0, T] \times[0, A] \cap\{s<t\} ; \\
D_{x} W_{0}, D_{s} W_{0} \text { belong to } C^{\alpha_{1}, \alpha_{2}}\left(\mathbb{R}^{N} \times[0, A]\right) ; \\
D_{x} W_{1}, D_{t} W_{1} \text { belong to } C^{\alpha_{1}, \alpha_{2}}\left(\mathbb{R}^{N} \times[0, T]\right) .
\end{gathered}
$$


Then there exists a unique solution of (25)-(27) such that

$$
\begin{gathered}
W \text { belongs to } C^{\alpha_{1}, \alpha_{2}, \alpha_{2}}\left(\mathbb{R}^{N} \times[0, T] \times[0, A]\right), \\
\text { and } W_{t}, W_{s}, D_{x} W \text { belong to } C^{\alpha_{1}, \alpha_{2}, \alpha_{2}}\left(N_{0}\right) ;
\end{gathered}
$$

furthermore, if

$$
\begin{gathered}
\left\|\left(\vec{b}, D_{x} \vec{b}\right)\right\|_{\alpha_{1}, \alpha_{2}} \leqslant \beta, \quad\left\|\left(W_{0}, D_{x} W_{0}, D_{s} W_{0}, D_{x} W_{1}, D_{t} W_{1} \|\right)\right\|_{\alpha_{1}, \alpha_{2}} \leqslant \gamma \\
\left\|\left(G, D_{x} G, D_{t} G, D_{s} G, D_{W} G\right)\right\|_{\alpha_{1}, \alpha_{2}, \alpha_{2}} \leqslant K \quad \text { at } W=W(x, t, s),
\end{gathered}
$$

then

$$
\left\|\left(D_{x} W, D_{t} W, D_{s} W\right)\right\|_{\alpha_{1}, \alpha_{2}, \alpha_{2}} \leqslant c_{1} \gamma+c_{2}(\beta, K) T
$$

where the last two norms are taken over $N_{0}, c_{2}(\beta, K)$ is a constant depending on $\beta, K$, but $c_{1}$ is independent of $\beta, K$.

The estimate (28) did not appear in [11] since it was not needed there; however this estimate is essential for the proof of Theorem 1.

Proof. It will be convenient to solve, instead of 25], the equation for $W(x, t, s)$ :

$$
W_{t}+W_{s}+b(x, t) \cdot \nabla_{x} W= \begin{cases}G_{1}(x, t, s, W) & \text { if } s>t, \\ G_{2}(x, t, s, W) & \text { if } s<t,\end{cases}
$$

with the same initial conditions (26), where

$$
G_{1}(x, t, s, W)=G(x, t, t+s, W), G_{2}(x, t, s, W)=G(x, t+s, s, W) .
$$

We note that the assumptions and assertions of the lemma remain valid if we replace 25 by $\left(25^{1}\right)$.

We introduce the characteristic curves with velocity $\vec{b}$ :

$$
\frac{\mathrm{d} X(x, l)}{\mathrm{d} l}=\vec{b}(X(x, l), l) \quad \text { for } l>0, X(x, 0)=x .
$$

Suppose $W$ is a solution of $\left(25^{1}\right)$. We can then express it as a solution of an ODE along the characteristic curves. Indeed, the function

$$
U_{1}(x, t, s)=W(X(x, t), t, t+s)
$$

satisfies, for fixed $s$,

$$
\frac{\mathrm{d} U_{1}}{\mathrm{~d} t}=G_{1}\left(x, t, s, U_{1}\right),\left.\quad U_{1}\right|_{t=0}=W_{0}(x, s),
$$

and the function

$$
U_{2}(x, t, s)=W(X(x, t+s), t+s, s)
$$

satisfies, for fixed $t$,

$$
\frac{\mathrm{d} U_{2}}{\mathrm{~d} s}=G_{2}\left(x, t, s, U_{2}\right),\left.\quad U_{2}\right|_{s=0}=W_{1}(x, t) .
$$


We denote by $\zeta=\zeta(\cdot, t)$ the inverse of the function $X=X(\cdot, t)$, i.e., $x=X(\zeta(x, t), t)$. Then $W(x, t, s)=U_{1}(\zeta(x, t), t, t+s)$ is a solution of $\left(25^{1}\right)$ for $t<s$. As in [11] one can prove that

$$
\left\|\frac{\partial \zeta}{\partial X}\right\|_{L^{\infty}} \leqslant C, \quad\left|\nabla_{x} \zeta(x, t)-\nabla_{x} \zeta(\bar{x}, \bar{t})\right| \leqslant C\left(|x-\bar{x}|^{\alpha_{1}}+|t-\bar{t}|^{\alpha_{2}}\right)
$$

where $C$ is a constant which depends only on $\beta$.

Using 29] we deduce that

$$
W_{x}=\frac{\partial U_{1}}{\partial \xi} \xi_{x} \in C^{\alpha_{1}, \alpha_{2}, \alpha_{2}}, \quad W_{s}=\frac{\partial U_{1}}{\partial s} \in C^{\alpha_{1}, \alpha_{2}, \alpha_{2}},
$$

and then also $W_{t}=G-\vec{b} \cdot \nabla_{x} W-W_{s}$ is in $C^{\alpha_{1}, \alpha_{2}, \alpha_{2}}$.

Similarly one can prove with the representation $U_{2}$ the existence and regularity of $W$ for $t>s$. Finally, the compatibility condition (27) implies that $U_{1}=U_{2}$ along the common characteristic curves initiating at any of the points $(\zeta, 0)$, and thus at any point $(x, t, t)$. Hence $W$ belongs to $C^{\alpha_{1}, \alpha_{2}, \alpha_{2}}\left(\mathbb{R}^{N} \times[0, T] \times[0, A]\right)$.

In order to prove the assertion 28 we note that

$$
\frac{\mathrm{d}}{\mathrm{d} t}\left(\frac{\partial U_{1}}{\partial \xi}\right)=\frac{\partial G_{1}}{\partial \xi}\left(\xi, t, s, U_{1}\right)+\frac{\partial G_{1}}{\partial U_{1}}\left(\xi, t, s, U_{1}\right) \frac{\partial U_{1}}{\partial \xi}
$$

so that, by 29],

$$
\begin{aligned}
\left|\frac{\mathrm{d}}{\mathrm{d} t}\left[\frac{\partial U_{1}}{\partial \xi}\left(\xi_{1}, t, s\right)-\frac{\partial U_{1}}{\partial \xi}\left(\xi_{2}, t, s\right)\right]\right| & \\
\leqslant & \left|\frac{\partial G_{1}}{\partial \xi}\left(\xi_{1}, t, s, U_{1}\left(\xi_{1}, t, s\right)\right)-\frac{\partial G_{1}}{\partial \xi}\left(\xi_{2}, t, s, U_{1}\left(\xi_{2}, t, s\right)\right)\right| \\
& +\left|\frac{\partial G_{1}}{\partial U_{1}}\left(\xi_{1}, t, s, U_{1}\left(\xi_{1}, t, s\right)\right) \frac{\partial U_{1}}{\partial \xi}\left(\xi_{1}, t, s\right)-\frac{\partial G_{1}}{\partial U_{1}}\left(\xi_{2}, t, s, U_{1}\left(\xi_{2}, t, s\right)\right) \frac{\partial U_{1}}{\partial \xi}\left(\xi_{2}, t, s\right)\right| .
\end{aligned}
$$

As in [11] one can show that the right-hand side is bounded by $\left|\xi_{1}-\xi_{2}\right|^{\alpha_{1}}$ times a constant which depends on $\beta, K$. Hence

$$
\left|\frac{\partial U_{1}}{\partial \xi}\left(\xi_{1}, t, s\right)-\frac{\partial U_{1}}{\partial \xi}\left(\xi_{2}, t, s\right)\right| \leqslant\left[c_{1} \gamma+c_{2}(\beta, K) T\right]\left|\xi_{1}-\xi_{2}\right|^{\alpha_{1}} .
$$

Similarly we can estimate

$$
\frac{\partial U_{1}}{\partial \xi}\left(\xi, t_{1}, s\right)-\frac{\partial U_{1}}{\partial \xi}\left(\xi, t_{2}, s\right)
$$

and all the other terms in 28 .

\section{Proof of Theorem 1}

We introduce the Banach space $Y$ of functions

$$
B \equiv\left\{p_{1}\left(x, t, A_{1}\right), p_{2}\left(x, t, A_{2}\right), p_{1}^{a}\left(x, t, A_{1}^{a}\right), p_{2}^{a}\left(x, t, A_{2}^{a}\right), \vec{Q}(x, t), \vec{Q}^{a}(x, t), w(x, t)\right\}
$$


with norm

$$
\|B\| \equiv\left\|\left(p_{1}, p_{2}, p_{1}^{a}, p_{2}^{a}, \vec{Q}, \vec{Q}^{a}\right)\right\|_{1+\alpha, \alpha / 3}+\|w\|_{\alpha, \alpha / 3}
$$

where $x \in \mathbb{R}^{N}, 0 \leqslant t \leqslant T$, and the subset

$$
Y_{M}=\{B \in Y: \text { the initial values are as in } 14, \text { and }\|B\| \leqslant M\}
$$

where $M$ is a positive constant to be determined.

For any $B \in Y_{M}$ we set

$$
h(x, t)=-H\left(\vec{Q}, \vec{Q}^{a}\right)
$$

and solve the inhomogeneous Hele-Shaw problem (22), 23) with $m=0$, thus generating a family of domains $\Omega(t)$ and a function $\sigma(x, t)$. We extend $\sigma(x, t)$ to a function $\sigma_{*}(x, t)$ in $\mathbb{R}^{N} \times[0, T]$ by continuing it along normals and using a fixed cutoff function, so that

$$
D_{x}^{2} \sigma_{*} \in C^{\alpha, \alpha / 3}\left(\mathbb{R}^{N} \times[0, T]\right) .
$$

Next we solve (10), (11) with initial data $w_{0}(x)$ and denote the solution by $w_{*}(x, t)$. We extend $w_{*}(x, t)$, in the same way as $\sigma(x, t)$, along normals with the same cutoff function, so that (cf. [11])

$$
D_{x}^{2} w_{*}, D_{t} w_{*} \in C^{2 \alpha / 3, \alpha / 3}\left(\mathbb{R}^{N} \times[0, T]\right) .
$$

We then proceed to solve $\sqrt{9}$ with $f_{i}=f_{i}\left(\vec{Q}, \vec{Q}^{a}, w_{*}\right)$ for $p_{i}(x, t, s), p_{i}^{a}(x, t, s), x \in \mathbb{R}^{N}, 0<$ $t<s_{i}(i=0,1,2,3)$ and for $p_{4}(x, t), p_{4}^{a}(x, t)$ using the initial conditions $[14)$; here we use the proof of Lemma 3.2 for $t<s$. We denote the solution by $p_{*_{i}}\left(x, t, s_{i}\right), p_{*_{i}}^{a}\left(x, t, s_{i}\right)(0 \leqslant i \leqslant 3)$, and $p_{*_{4}}(x, t), p_{*_{4}}^{a}(x, t)$. The functions $p_{*_{i}}\left(x, t, A_{i}\right), p_{*_{i}}^{a}\left(x, t, A_{i}^{a}\right)$ belong to $C^{1+\alpha, \alpha / 3}$. We proceed to solve the same system 9 for $t>s_{i}(i=0,1,2,3)$ using the data $p_{i}(x, t, 0), p_{i}^{a}(x, t, 0)$ (which are obtained by the relations 2 from $\left.p_{*_{i}}\left(x, t, A_{i}\right), p_{*_{i}}^{a}\left(x, t, A_{i}\right)\right)$ and the proof of Lemma 3.2 for $t>s$. In view of the compatibility condition (21), the functions $p_{*_{i}}, p_{*_{i}}^{a}$ are continuous across $t=s_{i}$ and thus they belong to $C^{\alpha_{1}, \alpha_{2}, \alpha_{2}}\left(\mathbb{R}^{N} \times[0, T] \times\left[0, A_{i}\right]\right)$ and $C^{\alpha_{1}, \alpha_{2}, \alpha_{3}}\left(\mathbb{R}^{N} \times[0, T] \times\left[0, A_{i}^{a}\right]\right)$, respectively.

From the functions $p_{*_{i}}, p_{*_{i}}^{a}$ we now construct the integrals

$$
Q_{*_{i}}=\int_{0}^{A_{i}} p_{*_{i}}\left(x, t, s_{i}\right) \mathrm{d} s_{i}, \quad Q_{*_{i}}^{a}=\int_{0}^{A_{i}^{a}} p_{*_{i}}^{a}\left(x, t, s_{i}\right) \mathrm{d} s_{i}
$$

and define a mapping $W$ by $W B=B_{*}$ where

$$
B_{*} \equiv\left\{p_{*_{1}}\left(x, t, A_{1}\right), p_{*_{2}}\left(x, t, A_{2}\right), p_{*_{1}}^{a}\left(x, t, A_{1}^{a}\right), p_{*_{2}}^{a}\left(x, t, A_{2}^{a}\right), \vec{Q}_{*}(x, t), \vec{Q}_{*}^{a}(x, t), w_{*}(x, t)\right\}
$$

and

$$
\vec{Q}_{*}=\left\{Q_{*_{i}}\right\}_{i=0}^{4}, \quad \vec{Q}_{*}^{a}=\left\{Q_{*_{i}}^{a}\right\}_{i=0}^{4}
$$

From the estimate 28 with $\vec{b}=-\nabla \sigma$ we deduce that

$$
\left\|\left(p_{*_{i}}, p_{*_{i}}^{a}\right)\right\|_{1+\alpha, 1+\alpha / 3,1+\alpha / 3} \leqslant c_{1} \gamma+c_{2}(\beta, M) T
$$

where the norm for $p_{*_{i}}$ is taken over

$$
N_{0 i}^{*}=\left\{\left(\mathbb{R}^{N} \times[0, T] \times\left[0, A_{i}\right]\right) \cap\left(t<s_{i}\right)\right\} \cup\left\{\left(\mathbb{R}^{N} \times[0, T] \times\left[0, A_{i}\right]\right) \cap\left(s_{i}<t\right)\right\}
$$


and the norm for $p_{*_{i}}^{a}$ is taken over the same set but with $A_{i}^{a}$; by Lemma 3.1 with $m=0$, we have $\beta \leqslant c_{3}(M)$.

Hence if

$$
M=c_{1} \gamma+1
$$

and $T$ is sufficiently small then $W$ maps $Y_{M}$ into itself.

We next show that $W$ is a contraction in the $L^{\infty}$-norm. To prove it take two elements $B_{1}$ and $B_{2}$ in $Y_{M}$ and set $W B_{i}=B_{*_{i}}$. We introduce the differences

$$
\hat{\sigma}=\sigma_{*_{1}}-\sigma_{*_{2}}, \quad \hat{w}=w_{*_{1}}-w_{*_{2}}
$$

corresponding to $B_{1}$ and $B_{2}$. As in [11],

$$
\|\hat{\sigma}\|_{1+\alpha,(1+\alpha) / 3} \leqslant c\left\|B_{1}-B_{2}\right\|_{L^{\infty}}, \quad\|\hat{w}\|_{1+\alpha,(1+\alpha) / 3} \leqslant c\left\|B_{1}-B_{2}\right\|_{L^{\infty}}
$$

where $c$ is a constant. By the arguments used to prove Lemma 3.2 we then obtain the estimate

$$
\left\|p_{* 1, i}-p_{* 2, i}\right\|_{\alpha, \alpha / 3} \leqslant c\left\|B_{1}-B_{2}\right\|_{L^{\infty}}
$$

where $p_{* 1, i}\left(x, t, A_{i}\right)-p_{* 2, i}\left(x, t, A_{i}\right)$ correspond to $B_{1}$ and $B_{2}$, respectively. Hence

$$
\left\|p_{* 1, i}-p_{* 2, i}\right\|_{L^{\infty}} \leqslant c T^{\beta}\left\|B_{1}-B_{2}\right\|_{L^{\infty}}
$$

for some $\beta>0$. The same inequality can be proved for the $p_{*_{i}}^{a}$. Hence, if $T$ is sufficiently small,

$$
\left\|W B_{1}-W B_{2}\right\|_{L^{\infty}}=\left\|B_{*_{1}}-B_{*_{2}}\right\|_{L^{\infty}} \leqslant c T^{\beta}\left\|B_{1}-B_{2}\right\|_{L^{\infty}} \leqslant \frac{1}{2}\left\|B_{1}-B_{2}\right\|_{L^{\infty}} .
$$

It follows that $W$ can have at most one fixed point.

Take any $B_{1} \in Y_{M}$. Then, by (32), the sequence $W^{n} B_{1}$ is convergent in the $L^{\infty}$-norm to some element $B$ in $Y_{M}$. It is also weakly convergent in the $Y$-norm. One can then easily show that $B$ is a fixed point of $W$. In order to complete the proof of Theorem 1, it remains to show that

$$
\sum_{i=0}^{4}\left(Q_{i}+Q_{i}^{a}\right)=1
$$

for the fixed point of $\mathrm{W}$. But this follows immediately from 15 and the easily derived relation

$$
\frac{\partial}{\partial t} \sum_{i=0}^{4}\left(Q_{i}+Q_{i}^{a}\right)=0 .
$$

REMARK 4.1 If the assumptions of Theorem 1 are satisfied for an integer $m>0$, then as in the proof of Theorem 1, Lemma 3.1 shows that

$$
\begin{gathered}
D_{\xi} D_{\xi, t}^{m} \rho \in C^{3+\alpha,(3+\alpha) / 3}\left(\Gamma_{0} \times[0, T]\right), \\
D_{x}^{2} D_{(x, t)}^{m} \sigma \in C^{\alpha, \alpha / 3}\left(\mathbb{R}^{N} \times[0, T]\right),
\end{gathered}
$$

and, by applying Lemma 3.2 step-by-step $m$ times,

$$
p_{i}, p_{i}^{a} \in C^{m+1+\alpha, m+\alpha / 3, m+\alpha / 3} \quad \text { for } t \neq s_{i} .
$$


In order to prove higher regularity of the $p_{i}, p_{i}^{a}$ across $t=s_{i}$ we need higher order compatibility conditions at $t=s_{i}=0$. We consider here just the second order compatibility conditions and, for clarity, we begin with the system (25), (26). We need to show that $W_{x}$ and $W_{s}$ are continuous across $t=s$. The function $W_{s}$ satisfies a hyperbolic equation similar to 25) with initial values, at $t=0$,

$$
\left.W_{s}\right|_{t=0}=W_{0, s}(x, s),
$$

and, from the differential equation $(25)$,

$$
\left.W_{s}\right|_{s=0}=\left[-W_{t}-\vec{b} \cdot \nabla_{x} W+G\right]_{s=0}=\left[-W_{1, t}-\vec{b} \cdot \nabla_{x} W+G\right]_{s=0} .
$$

Hence the compatibility condition is

$$
W_{1, t}+W_{0, s}+\vec{b} \cdot \nabla_{x} W_{0}=G\left(x, 0,0, W_{0}\right) \quad \text { at } t=s=0 .
$$

The compatibility condition for $W_{x}$ follows from (27).

Consider next the second compatibility conditions in the case of Theorem 1, and take for simplicity the case of $p_{1}$ at $(x, 0,0)$ and $p_{3}$ at $\left(x, A_{3}, 0\right)$. Then analogously to 33) we find that if

$$
\begin{aligned}
\frac{\partial p_{10}(x, 0)}{\partial s}-p_{10}(x, 0) f_{1}( & \left.\vec{Q}(x, 0), \vec{Q}^{a}(x, 0), w_{0}(x)\right) \\
& =\frac{\partial p_{30}\left(x, A_{3}\right)}{\partial s}-p_{30}\left(x, A_{3}\right) f_{3}\left(\vec{Q}(x, 0), \vec{Q}^{a}(x, 0), w_{0}(x)\right)
\end{aligned}
$$

then $\partial p_{1} / \partial s$ is continuous across $t=s_{1}$. The remaining second order compatibility conditions can similarly be written (but they have a more complicated form for $p_{2}$ and $p_{0}$ ). When all these conditions for both the $p_{i}$ and the $p_{i}^{a}$ are satisfied, then $p_{i}$ and $p_{i}^{a}$ will belong to $C^{2+\alpha, 1+\alpha / 3,1+\alpha / 3}$ across $t=s_{i}$.

\section{Radially symmetric solutions}

In this section, we consider the case when the initial data are radially symmetric, that is, in (14)

$$
\begin{aligned}
& \Omega_{0} \text { is a sphere of radius } R_{0}, \text { and } \\
& w_{0}=w_{0}(r), \quad p_{i 0}=p_{i 0}\left(r, s_{i}\right), \quad p_{i 0}^{a}=p_{i 0}^{a}\left(r, s_{i}\right)
\end{aligned}
$$

where $r=|x|$. We seek a solution of Problem (1)-15 which is radially symmetric in $x$, with

$$
\Omega(t)=\{r<R(t)\}
$$

In this special case we can relax the assumptions (19), 20) for $m=0$ by assuming that

$$
\text { the conditions (19), 20] hold with } C^{m+1+\alpha} \text { replaced by } C^{1} \text {. }
$$

We shall establish the existence and uniqueness of global-in-time solution. 
Set

$$
\begin{aligned}
& \Omega_{\infty}=\{(x, t):|x| \leqslant R(t), 0 \leqslant t<\infty\}, \\
& \Omega_{\infty}^{i}=\left\{\left(x, t, s_{i}\right) \in \Omega_{\infty} \times K_{i}: t \neq s_{i}+\sum_{j=0}^{3} n_{j} A_{j} \text { for any nonnegative integers } n_{j}\right\}, \\
& \Omega_{\infty}^{i a}=\left\{\left(x, t, s_{i}\right) \in \Omega_{\infty} \times K_{i}^{a}: t \neq s_{i}+\sum_{j=0}^{3} n_{j} A_{j} \text { for any nonnegative integers } n_{j}\right\} .
\end{aligned}
$$

THEOREM 2 Under the assumptions (35), (36), and (21) there exists a unique radially symmetric solution of Problem (1)-15) for all $t>0$, with $R(t)$ in $C^{1}[0, \infty),\left(D_{x} \sigma, w\right)$ in $C^{1}\left(\Omega_{\infty}\right), p_{i}$ in $C\left(\Omega_{\infty} \times K_{i}\right), \partial p_{i} / \partial r$ and $\partial p_{i} / \partial s$ in $C\left(\Omega_{\infty}^{i}\right), p_{i}^{a}$ in $C\left(\Omega_{\infty} \times K_{i}^{a}\right)$, and $\partial p_{i}^{a} / \partial r$ and $\partial p_{i}^{a} / \partial s$ in $C\left(\Omega_{\infty}^{i a}\right)$.

REMARK 5.1 In order to explain why the discontinuities of the first derivatives of $p_{i}\left(r, t, s_{i}\right)$ are included in the set $t=s_{i}+\sum_{j=0}^{3} n_{j} A_{j}$ where $n_{j}$ are nonnegative integers, consider a special case where $A_{3}<A_{1}$ and $A_{1}<A_{0}, A_{1}<A_{2}$. For $t<A_{1}$ the discontinuities of the first derivatives of $p_{1}\left(r, t, s_{1}\right)$ can occur either at $t=s_{1}$ or at $t=s_{1}+A_{3}$ due to the relation $p_{1}(r, t, 0)=p_{3}\left(r, t, A_{3}\right)$. For $t>s_{1}$ but $t-s_{1}$ small, discontinuities may occur at $t=s_{1}+A_{1}$ and $t=s_{1}+A_{3}$. As $t$ increases new discontinuous branches may be introduced because of the relations (2), but they are all of the form $t=s_{i}+\sum_{j=0}^{3} n_{j} A_{j}$.

Proof of Theorem 2. Note that in the radially symmetric case

$$
\begin{aligned}
& \vec{v}=\frac{x}{r} u(r, t), \\
& \operatorname{div}(p \vec{v})=u \frac{\partial p}{\partial r}+p \frac{1}{r^{N-1}} \frac{\partial}{\partial r}\left(r^{N-1} u\right) \quad \text { if } p=p(r), \\
& u(r, t)=\frac{1}{r^{N-1}} \int_{0}^{r} r^{N-1} H\left(\vec{Q}, \vec{Q}^{a}, w\right) \mathrm{d} r,
\end{aligned}
$$

and the free boundary condition is

We introduce a change of variables

$$
\frac{\mathrm{d} R}{\mathrm{~d} t}=u(R(t), t)
$$

$$
\begin{gathered}
\tilde{r}=\frac{r}{R(t)}, \quad \tilde{p}_{i}\left(\tilde{r}, t, s_{i}\right)=p_{i}\left(r, t, s_{i}\right), \quad \tilde{p}_{i}^{a}\left(\tilde{r}, t, s_{i}\right)=p_{i}^{a}\left(r, t, s_{i}\right), \\
\tilde{w}(\tilde{r}, t)=w(r, t) \quad \text { and } \quad \tilde{u}(\tilde{r}, t)=\frac{u(r, t)}{R(t)} .
\end{gathered}
$$

Then the system $[1]-15$ is transformed into a new system in the fixed domain $\{\tilde{r}<1\}$. Dropping the tildes in the above variables, the new PDE system takes the following form:

$$
\begin{array}{cc}
\frac{\partial p_{i}}{\partial t}+\frac{\partial p_{i}}{\partial s_{i}}+v \frac{\partial p_{i}}{\partial r}=p_{i} f_{i}\left(\vec{Q}, \vec{Q}^{a}, w\right) & (0 \leqslant i \leqslant 3), \\
\frac{\partial p_{i}^{a}}{\partial t}+\frac{\partial p_{i}^{a}}{\partial s_{i}}+v \frac{\partial p_{i}^{a}}{\partial r}=p_{i} f_{i}^{a}\left(\vec{Q}, \vec{Q}^{a}, w\right) & (0 \leqslant i \leqslant 3),
\end{array}
$$




$$
\frac{\partial p_{4}}{\partial t}+v \frac{\partial p_{4}}{\partial r}=\mu_{1} p_{1}\left(r, t, A_{1}\right)+\mu_{2} p_{2}\left(r, t, A_{2}\right)+p_{4} f_{4}\left(Q, \vec{Q}^{a}, w\right)
$$

where

$$
\begin{gathered}
v(r, t)=u(r, t)-r u(1, t), \\
w_{t}-\frac{r}{R} \dot{R} w_{r}-\frac{1}{R^{2}} D_{w} \nabla^{2} w+\lambda Q w=0 .
\end{gathered}
$$

The initial and boundary conditions have the same form as before, and the free boundary condition is

$$
\frac{\mathrm{d} R}{\mathrm{~d} t}=R(t) u(1, t)
$$

where $u$ is given by (37).

Analogously to the proof of Theorem 1 we introduce the Banach space $Y$ of functions

$$
B=\left\{p_{1}\left(r, t, A_{1}\right), p_{2}\left(r, t, A_{2}\right), p_{1}^{a}\left(r, t, A_{1}^{a}\right), p_{2}^{a}\left(r, t, A_{2}^{a}\right), \vec{Q}(r, t), \vec{Q}^{a}(r, t), w(r, t), R(t)\right\}
$$

for $0 \leqslant t \leqslant T$, with norm

$$
\|B\|=\left\|\left(p_{1}, p_{2}, p_{1}^{a}, p_{2}^{a}, \vec{Q}, \vec{Q}^{a}\right)\right\|_{0}+\|w\|_{0}+\|(R, \dot{R})\|_{0}
$$

where the uniform norm \|\|$_{0}$ is taken over $0 \leqslant r \leqslant 1,0 \leqslant t \leqslant T$.

Let

$$
Y_{M}=\left\{B \in Y \text { with initial data as in 34), }\|B\| \leqslant M, R(0)=R_{0}\right\}
$$

where $M$ is to be determined. If $T$ is sufficiently small then $R(t) \geqslant$ const $>0$.

The characteristic curves of $38-40$ are given by

$$
\frac{\mathrm{d} r}{\mathrm{~d} t}=v(r, t), \quad \text { and } \quad \frac{\partial v}{\partial r} \text { is bounded. }
$$

If $t<s_{i}$ then a characteristic curve, when extended backward in time, arrives, at $t=0$, at some point $\left.r\right|_{t=0}=\xi$, where $\xi \in(0,1)$. If we denote such a characteristic curve by $r(\xi, t)$, then

$$
\frac{\partial r(\xi, t)}{\partial \xi}=\exp \left\{\int_{0}^{t} \frac{\partial v}{\partial r}(r(\xi, \tau), \tau) \mathrm{d} \tau\right\} .
$$

For any element $B$ in $Y_{M}$ we solve the system for $p_{i}, p_{i}^{a}, w$ by using Lemma 3.2, and denote the solution by $p_{*_{i}}, p_{*_{i}}^{a}, w_{*}$. In accordance with 37 we define

$$
u_{*}(r, t)=\frac{1}{r^{N-1}} \int_{0}^{r} r^{N-1} H\left(\vec{Q}_{*}, \vec{Q}_{*}^{a}, w_{*}\right) \mathrm{d} r
$$

where $\vec{Q}_{*}, \vec{Q}_{*}^{a}$ are defined in terms of the $p_{*_{i}}, p_{*_{i}}^{a}$ as in the proof of Theorem 1 . We also define $R_{*}(t)$ by (43) with $u=u_{*}$, that is,

$$
R_{*}(t)=R_{0} \exp \left(\int_{0}^{t} u_{*}(1, \tau) \mathrm{d} \tau\right) .
$$


As in the proof of Lemma 3.2 we can derive uniform estimates on $\partial p_{*_{i}} / \partial r, \partial p_{*_{i}} / \partial s_{i}$ and $\partial p_{*_{i}}^{a} / \partial r, \partial p_{*_{i}}^{a} / \partial s_{i}$ for $t<s_{i}$, and then also for $s_{i}<t$, and $p_{*_{i}}, p_{*_{i}}^{a}$ are continuous at $t=s_{i}$ by the compatibility assumption (21). Thus

$$
\left\|\left(\frac{\partial p_{*_{i}}}{\partial r}, \frac{\partial p_{*_{i}}^{a}}{\partial r}, \frac{\partial p_{*_{i}}}{\partial s_{i}}, \frac{\partial p_{*_{i}}^{a}}{\partial s_{i}}\right)\right\|_{0}^{*} \leqslant \bar{c}_{1}+\bar{c}_{2} T
$$

where the upper star " $*$ " indicates that the uniform norm is taken separately over $t<s_{i}$ and over $t>s_{i} ; \bar{c}_{1}, \bar{c}_{2}$ are constants which depend on a uniform bound on the initial data, and $\xi$ depends also on $M$.

Setting

$B_{*}=\left\{p_{*_{1}}\left(r, t, A_{1}\right), p_{*_{2}}\left(r, t, A_{2}\right), p_{*_{1}}^{a}\left(r, t, A_{1}^{a}\right), p_{*_{2}}^{a}\left(r, t, A_{2}^{a}\right), \vec{Q}_{*}(r, t), \vec{Q}_{*}^{a}(r, t), w_{*}(r, t), R_{*}(t)\right\}$ we define a mapping $W$ by $W B=B_{*}$.

From (47) we deduce that if $M$ is large enough, depending on the initial data, then, for $T$ small enough, $W$ maps $Y_{M}$ into itself.

One can also show by the same argument as in [12; Section 3] that $W$ is a contraction if $T$ is sufficiently small. We conclude that there exists a unique radially symmetric solution of Problem (1)-(15) for $0 \leqslant t \leqslant T, T$ small.

In order to prove global-in-time existence we need to prove some a priori estimates. Assuming that a solution exists for some time interval $0 \leqslant t<T$, where $T$ is any positive number, all we need to prove is that

$$
0<c_{1} \leqslant R(t), \quad\left|\frac{\mathrm{d} R}{\mathrm{~d} t}\right| \leqslant c_{2} \quad \text { for } 0 \leqslant t<T
$$

and that

$$
\begin{aligned}
\left\|\left(p_{i}, \frac{\partial p_{i}}{\partial r}, \frac{\partial p_{i}}{\partial s}\right)\right\|_{0, \Omega_{\infty}^{i} \cap\{t<T\}}+\left\|\left(p_{i}^{a}, \frac{\partial p_{i}^{a}}{\partial r}, \frac{\partial p_{i}^{a}}{\partial s}\right)\right\|_{0, \Omega_{\infty}^{i a} \cap\{t<T\}} & +\left\|\left(w, w_{r}\right)\right\|_{0, \Omega_{\infty} \cap\{0 \leqslant t<T\}} \leqslant c_{3}
\end{aligned}
$$

where the $c_{i}$ are positive constant (which may depend on $T$ ). Indeed, the $M$ used in the proof of local existence will then remain uniformly bounded in $\varepsilon$ if we repeat the proof starting at any time $t=T-\varepsilon, \varepsilon>0$, and thus the solution can be extended to some interval $0 \leqslant t \leqslant T+\varepsilon_{0}, \varepsilon_{0}>0$.

Since $Q$ is uniformly bounded it follows by the maximum principle that $w$ is uniformly bounded. From (37) and the boundedness of the $Q_{i}, Q_{i}^{a}$ we also deduce that

$$
\left\|\frac{\partial v}{\partial r}\right\|_{0} \leqslant c_{4} \quad \text { for } 0 \leqslant t<T .
$$

Since $u$ is also bounded, 43 gives

$$
-c_{5} \leqslant \frac{1}{R} \frac{\mathrm{d} R}{\mathrm{~d} t} \leqslant c_{6}
$$

and (48) follows. Using (48) and $L^{p}$ estimates in (41) we deduce that $w_{r}$ is uniformly bounded as claimed in 49 . We next use the representation of $p_{i}$ by means of characteristic curves as in 29], (30) to deduce, using the form of equations (9), that

$$
\left\|p_{i}\right\|_{0}+\left\|p_{i}^{a}\right\|_{0} \leqslant c_{5} \quad\left(\text { actually } c_{5}=C_{0} c^{c_{0} T} \text { where } C_{0}, c_{0} \text { are independent of } T\right. \text { ). }
$$


Differentiating these equations in $s_{i}$ we obtain similar estimates for $\partial p_{i} / \partial s$ if $t \neq s_{i}+\sum_{j=0}^{3} n_{j} A_{j}$ where $n_{j}$ are nonnegative integers, and similar estimates for $\partial p_{i}^{a} / \partial s$ if $t \neq s_{i}+\sum_{j=0}^{3} n_{j} A_{j}$. Similarly we estimate $\partial p_{i} / \partial r, \partial p_{i}^{a} / \partial r$ using the fact that

$$
\left|\frac{\partial Q_{i}(r, t)}{\partial r}\right| \leqslant A_{i} \sup _{s_{i}}\left|\frac{\partial p_{i}\left(r, t, s_{i}\right)}{\partial r}\right|, \quad\left|\frac{\partial Q_{i}^{a}(r, t)}{\partial r}\right| \leqslant A_{i}^{a} \sup _{s_{i}}\left|\frac{\partial p_{i}^{a}\left(r, t, s_{i}\right)}{\partial r}\right| .
$$

This completes the proof of Theorem 2.

We conclude this section with some open problems.

Open Problems 1. In the case where there are only three types of cells, proliferating, quiescent and dead, it was proved in [13] that the global solution satisfies

$$
0<\delta \leqslant R(t) \leqslant A<\infty \text { for all } t>0 .
$$

Under what conditions on the $\lambda_{i}(w), \lambda_{i}^{a}(w)$ and $\lambda_{4}, \lambda_{4}^{a}$ are these inequalities valid?

2. In the case where there are only proliferating and dead cells, it was proved in [14] that there exists a unique stationary solution, and it was shown in [10] that this solution is linearly stable. Can such results be proved for the present model?

3. Consider Problem (1)- 15$)$ in the case when there are no mutations and denote the free boundary by $r=R_{S}(t)$. Prove that $R(t)>R_{S}(t)$.

\section{Acknowledgments}

This work is partially supported by the National Science Foundation, Division of Mathematical Sciences, upon Agreement 0112050.

\section{REFERENCES}

1. Aranjo, R. P., \& MCElwain, D. L. S. A history of the study of solid tumor growth: The contribution of mathematical modelling. Bull. Math. Biol. 66 (2003), 1039-1091.

2. AyAti, B. P., WebB, G. F., \& Anderson, A. R. A. Computational methods and results for structured mutliscale methods of tumor invasion. Multiscale Model. Simul. 5 (2006), 1-20. Zbl pre05037906 MR 2221307

3. BYRne, H. M. The importance of intercellular adhesion in the development of carcinomas. IMA J. Math. Appl. Med. Biol. 14 (1997), 305-323. Zbl 0891.92017

4. BYRne, H. M. A weakly nonlinear analysis of a model of vascular solid tumor. J. Math. Biol. 39 (1999), 59-89. Zbl 0981.92011 MR 1705626

5. B YRne, H. M., \& Chaplain, M. A. J. Growth of nonnecrotic tumors in the presence and absence of inhibitors. Math. Biosci. 130 (1995), 151-181. Zbl 0836.92011

6. ByRne, H. M., \& CHAPLAin, M. A. J. Growth of necrotic tumors in the presence and absence of inhibitors. Math. Biosci. 135 (1996), 187-216. Zbl 0856.92010

7. Byrne, H. M., \& Chaplain, M. A. J. Modelling the role of cell-cell adhesion in the growth and development of carcinomas. Math. Comput. Modeling 24 (1996), 1-17. Zbl 0883.92014

8. Byrne, H. M., \& Chaplain, M. A. J. Free boundary value problems associated with growth and development of multicellular spheroids. Eur. J. Appl. Math. 8 (1997), 639-658. Zbl 0906.92016 MR 1608619 
9. Chaplain, M. A. J. The development of a spatial pattern in a model for cancer growth. In: H. G. Othmer et al. (eds.), Experimental and Theoretical Advances in Biological Pattern Formation, Plenum Press (1993), 45-60.

10. Chen, X., Cui, S., \& Friedman A. A hyperbolic free boundary problem modeling tumor growth: Asymptotic behavior. Trans. Amer. Math. Soc. 357 (2005), 4771-4804. Zbl 1082.35166 MR 2165387

11. Chen, X., \& Friedman, A. A free boundary problem for elliptic-hyperbolic system: An application to tumor growth. SIAM J. Math. Anal. 35 (2003), 974-986. Zbl 1054.35144 MR 2049029

12. Chen, X., Hong, J., \& YI, F. Existence, uniqueness, and regularity of classical solutions of the Mullins-Sekerka problem. Comm. Partial Differential Equations 21 (1996), 1705-1727. Zbl 0884.35177 MR 1421209

13. CUI, S., \& FRIEDmAn, A. A hyperbolic free boundary problem modeling tumor growth. Interfaces Free Bound. 5 (2003), 159-182. Zbl 1040.35143 MR 1980470

14. CUI, S., \& FRIEDMAN, A. A free boundary problem for a singular system of differential equations: An application to a model of tumor growth. Trans. Amer. Math. Soc. 355 (2003), 3537-3590. Zbl 1036.34018 MR 1990162

15. Fontelos, M. A., \& Friedman, A. Symmetry-breaking bifurcations of free boundary problems in three dimensions. Asymptotic Anal. 35 (2003), 187-206. Zbl 1054.35145 MR 2011787

16. Friedman, A. Mathematical analysis and challenges arising from models of tumor growth. Math. Models Methods Appl. Sci. 17 (2007), 1751-1772. MR 2362763

17. Friedman, A., \& Hu, B. Bifurcation from stability to instability for a free boundary problem arising in tumor model. Arch. Ration. Mech. Anal. 180 (2006), 293-330. Zbl 1087.92039 MR 2210911

18. Friedman, A., \& Hu, B. Asymptotic stability for a free boundary problem arising in a tumor model. J. Differential Equations 227 (2006), 598-639. Zbl pre05047166 MR 2237681

19. Friedman, A., \& Reitich, F. Analysis of a mathematical model for growth of tumors. J. Math. Biol. 38 (1999), 262-284. Zbl 0944.92018 MR 1684873

20. Friedman, A., \& ReITICH, F. Symmetry-breaking bifurcation of analytic solutions to free boundary problems: An application to a model of tumor growth. Trans. Amer. Math. Soc. 353 (2001), 1587-1634. Zbl 0983.35019 MR 1806728

21. Greenspan, H. Models for the growth of solid tumor by diffusion. Stud. Appl. Math. 51 (1972), 317340. Zbl 0257.92001

22. GreEnspan, H. On the growth and stability of cell cultures and solid tumors. J. Theoret. Biol. 56 (1976), 229-242. MR 0429164

23. Pettet, G. J., Please, C. P., Tindall, M. J., \& McElwain, D. L. S. The migration of cells in multicell tumor spheroids. Bull. Math. Biol. 63 (2001), 231-257. MR 2224767

24. RiBBA, B., Colin, T., \& SChnell, S. A multiscale model of cancer, and its use in analyzing irradiation therapies. Theoret. Biology Medical Modeling 3 (2006), no. 7, 1-19. 